\title{
Birth of the INR
}

Since the first recorded clinical use of coumarin as an anticoagulant agent in 1941 (MILESTONE 2), vitamin K antagonists (VKAs) have been the mainstay of anticoagulation therapy for $>50$ years. Today, warfarin remains the most frequently prescribed oral anticoagulant for the treatment of venous thromboembolism and prevention of stroke in patients with atrial fibrillation. However, early use of VKAs was fraught with challenges related to variations in an individual's response to the drug, because the efficacy and safety of the drug greatly depend on maintaining the anticoagulant effect within a defined therapeutic range.

Prothrombin time (PT), developed by Armand Quick and colleagues in 1935 , is a measure of the time required for plasma to clot after addition of a thromboplastin reagent, and was the earliest basis for oral anticoagulant monitoring. This measure had substantial drawbacks, however, such as the conflicting PT results from different laboratories, and inconsistent methods of data reporting (time, ratio, or percentage activity). Furthermore, thromboplastin reagents made in-house (with human brain extracts) and commercially (from animal tissues) differed in their sensitivity to reductions in vitamin K-dependent clotting factors.

In the 1960s in North American

laboratories, sensitive in-house reagents were swapped for less-sensitive commercial reagents, which undoubtedly would have influenced drug dosing. Unfortunately, these discrepancies were not recognized until 1982 when Leon Poller and David Taberner published an international survey of warfarin dosage, which reported higher doses of warfarin administration in North America where laboratories were still using less-sensitive commercial thromboplastins, and lower warfarin doses prescribed in centres in the UK that prepared thromboplastins in-house from human brain extracts. In the same year, Russell Hull and colleagues published a randomized study comparing patients with proximal vein thrombosis treated with a dose of warfarin adjusted according to a PT reagent made with animal tissues (commonly used in North America) to those treated with a dose of warfarin adjusted according to a PT reagent made with human brain thromboplastin (commonly used in the UK). The findings from the study showed that significantly larger doses of warfarin were prescribed in North America, despite lower target PT ratios. Importantly, both groups of patients were protected from current venous thrombosis, but patients treated with the UK regimen had significantly fewer bleeding complications.

In 1977, recognizing the need for a standardized measure of PT, the Expert Committee on Biological Standardization of the WHO proposed a scheme for calibrating thromboplastins in terms of an International Reference Preparation. In 1983, Tom Kirkwood from the UK National Institute for Biological Standards and Control further developed this model to improve its suitability for calibrating thromboplastins of different types and species. The WHO Expert Committee approved this revised scheme in the same year, giving rise to the International Normalized Ratio (INR), a measure still used today to monitor patients taking VKA therapy.

Despite its obvious advantages over PT, adoption of the INR within the clinical community was initially slow. However, by 1995, most laboratories in the USA were reporting INR values, and all manufacturers of thromboplastin reagents were required to provide an international sensitivity index for their reagent, which is needed to calculate the INR. ce

Early use of

VKAs was

fraught with challenges related to variations in an individual's response to the drug g5
The potential benefits versus harms of VKA therapy rely on good control of anticoagulation intensity within a narrow INR range. At present, the optimal therapeutic range for oral anticoagulation is considered to be an INR of 2.0-3.0 for patients at risk of recurrent venous thromboembolism or patients with atrial fibrillation with an intermediate-to-high risk of stroke. However, the INR should be maintained between 2.5 and 3.5 in patients with mechanical prosthetic heart valves. The beneficial effect of VKA therapy on thromboembolic risk was confirmed in 2003 in a study by Go and colleagues, who reported that VKAs reduced the risk of ischaemic stroke and peripheral thromboembolism in a large cohort of ambulatory patients with atrial fibrillation, consistent with results reported from randomized trials. Although VKAs are being gradually supplanted by newer non-vitamin $\mathrm{K}$ antagonist oral anticoagulants (MILESTONE 10), VKAs still remain the drug of choice for patients with atrial fibrillation and rheumatic valvular disease, and for patients with mechanical heart valves.

Karina Huynh, Senior Editor, Nature Reviews Cardiology

ORIGINAL ARTICLES Poller, L. \& Taberner, D. A. Dosage and control of oral anticoagulants: an international survey. Br.J. Haematol. 51, 479-485 (1982)| Hull, R. et al. Different intensities of oral anticoagulant therapy in the treatment of proximalvein thrombosis. N. Engl.J. Med. 307, 1676-1681 (1982) | Go, A. S. et al. Anticoagulation therapy for stroke prevention in atrial fibrillation: how well do randomized trials translate into clinical practice? JAMA 288, 2441-2448 (2003) FURTHER READING Hirsh, J. \& Poller, L. The international normalized ratio. A guide to understanding and correcting its problems. Arch. Intern. Med. 154, 282-288 (1994)| Hirsh, J. Solving the mystery of excessive warfarin-induced bleeding: a personal historical perspective. Thromb. Haemost. 112, 853-856 (2014) 